DOI: https://doi.org/10.11144/Javeriana.upsy18-3.rspu

\title{
Riesgos para la salud de profesores universitarios derivados de factores psicosociales laborales*
}

\section{Health Risk for University Professors Associated to Occupational Psychosocial Risk Factors}

\author{
Viviola Gómez Ortiz a \\ Universidad de los Andes, Colombia \\ ORCID: http://orcid.org/0000-0002-1066-9483 \\ Lyria Esperanza Perilla-Toro \\ Universidad de los Andes, Colombia \\ ORCID: http://orcid.org/0000-0002-0856-456X \\ Angélica María Hermosa R. \\ Universidad Central, Colombia \\ ORCID: http://orcid.org/0000-0002-9101-4664
}

a Autor de correspondencia. Correo electrónico: vgomez@uniandes.edu.co

Para citar este artículo: Gómez Ortiz, V., Perilla-Toro, L. E., \& Hermosa, A. M. (2019). Riesgos para la salud de profesores universitarios derivados de factores psicosociales laborales. Universitas Psychologica, 18(3), 1-15. https://doi.org/10.11144/Javeriana.upsy18-3. rspu

\section{RESUMEN}

El propósito del estudio fue identificar factores de riesgo psicosocial ocupacional en académicos de universidades de Bogotá y establecer si están asociados o no con diversos indicadores de malestar psicológico. En el estudio, participaron 302 académicos de 21 universidades de la ciudad. Los resultados evidenciaron que la inseguridad laboral, las excesivas exigencias laborales y la alta carga de trabajo son los principales factores psicosociales laborales generadores de estrés en los profesores universitarios. Quienes más perciben demandas y carga de trabajo son también quienes más reportan malestar de diverso tipo, tales como ansiedad, síntomas psicosomáticos y desajuste social. Las demandas, exigencias y esfuerzos laborales son percibidos como más altos por parte de los profesores más jóvenes, con categoría de asistentes. Los resultados de este estudio señalan la necesidad de las universidades de revisar sus prioridades y definir cómo aprovechar de la mejor manera el potencial de su principal capital que son los académicos, sin que estos terminen lastimados por sus esfuerzos y compromiso con las instituciones.

Palabras clave

factores de riesgo psicosociales; profesores universitarios; malestar psicológico; modelo demanda-control; modelo esfuerzo-recompensa.

\begin{abstract}
The purpose of the study was to identify occupational psychosocial risk factors of academics from universities in Bogotá and establish if they are related with indicators of psychological distress. 302 professors from 21 universities in the city participate in the study. The results showed that job insecurity, excessive work demands, and high workload are the main psychosocial factors generating the stress of university professors. Those who perceive more demands and workload are also those who most report distress problems such as anxiety, psychosomatic symptoms, and social maladjustment. The demands and work efforts are perceived as higher by
\end{abstract}


the younger, assistant professors. The results of this study point out the need for universities to review their priorities and define how to make use of the potential of their main capital, the academics, without them being harmed by their efforts and commitment to the institutions.

Keywords

psychosocial risk factors; faculty; psychological distress; jobdemand model; effort-reward model.

El propósito de esta investigación fue identificar los Factores de Riesgo Psicosocial (FRP) presentes en el contexto laboral de profesores universitarios en Bogotá (Colombia) y establecer si están asociados o no con diversos indicadores de salud. Este propósito es importante dadas las condiciones de cambio a las que el sistema educativo colombiano ha estado sometido en los últimos años, y en el contexto de una reglamentación nacional que reconoce la necesidad de identificar, evaluar, prevenir, intervenir y monitorear permanentemente la exposición a FRP en el trabajo y su impacto en la salud (Resolución 2646 de 2008 del Ministerio de la Protección Social de Colombia).

Gran parte de los cambios en el sistema educativo mundial se debe a que el entorno social (Estado, padres, alumnos, pares) impone al ambiente educativo cada vez más exigencias y modificaciones tales como la internacionalización curricular, la movilidad docente, la construcción de redes, las investigaciones conjuntas, el compartir buenas prácticas educativas, las restricciones financieras, nuevas regulaciones atadas a la búsqueda de la calidad, entre otras (Doménech, 1995; Instituto Internacional de la Unesco para la Educación Superior en América Latina y el Caribe, 2018). Estas exigencias y cambios pueden ser positivos, sin embargo, también representan un aumento importante de demandas laborales de tipo psicosocial (p. ej., más presiones de tiempo, exigencias de mayor publicación). Así mismo, los docentes podrían percibir que sus habilidades previas no son suficientes para responder a los nuevos retos y que se limitan sus posibilidades para decidir cómo y cuándo desempeñar su trabajo. Estas condiciones no solo menoscaban la salud física y el bienestar psicológico de los profesores, también los de sus alumnos. Por último, la organización también puede afectarse por aspectos tales como ausentismo, mal desempeño, abandono de la actividad laboral, disminución de la calidad del trabajo, baja satisfacción laboral, entre otros (Heredia et al., 2018).

Diversos estudios (p. ej., Cross \& Carroll, 1990; Doyle \& Hind, 2002; García, Iglesias, Saleta, \& Romay, 2016; Jackson \& Hayday, 1997; Keser, Li, \& Siegrist, 2019; Kinman, 1998, 2001; Kinman, Jones, \& Kinman, 2006; McInnis, 1999; Winefield \& Jarrett, 2001) realizados en España, Inglaterra, Estados Unidos, Australia y Turquía señalan la inseguridad laboral, el excesivo número de horas de trabajo a la semana y las demandas o exigencias laborales, tales como sobrecarga en tiempos para la supervisión de estudiantes y el requerimiento permanente de docencia y el trabajo de investigación en tiempo extra, como los principales factores psicosociales laborales generadores de estrés de los académicos universitarios. También se reportan como estresores laborales: presión por obtener dinero para investigación, tiempo insuficiente para estar al tanto de los desarrollos en las áreas de experticia, salarios inadecuados, frecuentes interrupciones en el trabajo, conflicto entre los roles y lento desarrollo de carrera.

En países latinoamericanos, también se han evaluado e identificado condiciones psicosociales de riesgo asociadas al trabajo en las universidades (Collado, Soria, Canafoglia, \& Collado, 2016; Heredia et al., 2018; Lemos et al., 2019; Pando, Aranda, Aldrete, Flores, \& Pozos, 2006; Pando, Castañeda et al., 2006; Pujol-Cols \& Arraigada, 2017; Rodríguez et al., 2018; Unda et al., 2016). Estos estudios reportaron que los factores psicosociales negativos de mayor prevalencia fueron los relacionados con las interacciones personales que se derivan del trabajo y la esencia de la tarea, los del sistema laboral y los propiamente organizacionales. Las exigencias laborales que se reportaron fueron: requerir constantemente creatividad e iniciativa, tener que hablar frecuentemente, enseñar a personas que no valoran la educación, necesitar continuamente un alto 
grado de concentración, falta de reconocimiento, percepción de inequidad y de falta de recursos, interferencia de asuntos laborales en la familia y viceversa, además de tener que mostrar buenos resultados simultáneamente en diferentes tipos de tareas (docencia, investigación, consultoría y gestión).

Las condiciones arriba descritas se han estudiado, en su mayor parte, sin tomar en cuenta un marco teórico específico. Sin embargo, estos factores psicosociales laborales podrían conceptualizarse con base en los principales modelos de estrés laboral existentes en la literatura: el Modelo Demanda-Control-Apoyo Social (Karasek \& Theorell, 1990) ${ }^{1}$ y el Modelo Desequilibrio Esfuerzo-Recompensa ${ }^{2}$ (Siegrist, 2002). Ambos modelos proponen que la presencia crónica de ciertas condiciones laborales, descritas en cada modelo, aumenta el riesgo de activación psicobiológica prolongada y, por tanto, de consecuencias negativas para la salud (Sauter, Murphy, Hurrell, \& Levi, 1998; Theorell \& Johnson, 1998). Schnall, Belkic, Landsbergis y Baker (2000), además de Siegrist (2002) y Theorell y Johnson (1998) afirman que numerosos estudios epidemiológicos han presentado resultados que apoyan las hipótesis de estos modelos, en el sentido de que una exposición crónica a los FRP en el campo laboral aumenta el riesgo de desarrollar enfermedades de tipo cardiovascular. La salud mental que se ve afectada por estos factores, también se ha estudiado ampliamente (Van Der Doef \& Maes, 1999). La revisión de la literatura arrojó pocos trabajos con profesores universitarios, que utilizaran alguno de estos modelos conceptuales para evaluar los FRP en este grupo ocupacional (p. ej., García et al., 2016; Keser et al., 2019; Kinman \& Jones, 2003; Mark \& Smith, 2012; McClenahan, Giles, \& Mallett, 2007).

Además de no utilizar modelos conceptuales, se encontró que muchos de los estudios previamente citados describen condiciones psicosociales de riesgo, pero no las asocian con diversos estados de salud. Los que han establecido esta relación demuestran que no es directa, sino moderada por algunas variables tales como la experiencia y la edad. Por ejemplo, Winefield y Jarrett (2001), estudiando profesores australianos, encontraron que un aspecto interesante de sus hallazgos fue que, a pesar de los altos niveles de trastornos psicológicos identificados dentro de esta población, la satisfacción laboral fue relativamente alta y que los niveles de estrés fueron más altos en personal académico más joven y en aquellos con responsabilidades tanto docentes como de investigación; igualmente, se reportó aumento en satisfacción laboral y disminución en ansiedad con la antigüedad académica, es decir, a mayor antigüedad mayor satisfacción laboral y menor ansiedad. En cuanto a trastornos psicológicos percibidos, estos fueron bajos en los profesores con estatus altos y bajos, y en los niveles intermedios, se encontró el promedio más alto de trastornos. La investigación realizada en España por Cifre y Llorens (2001) identificó que los profesores-ayudantes, es decir los más jóvenes y con menos estabilidad laboral, son los que más riesgos psicosociales muestran por la presencia de altos niveles de sobrecarga emocional y cuantitativa, mayores niveles de burnout (agotamiento emocional y cinismo), ansiedad y depresión, seguidos de los profesores asociados de tiempo completo, quienes, en ese país, tampoco poseen un cargo que les garantice estabilidad laboral.

En conclusión, los trabajos realizados con profesores universitarios confirman la necesidad de más investigaciones que establezcan relaciones entre las condiciones laborales y la salud de los profesores, usando modelos teóricos sobre el estrés laboral, midiendo variables de salud y evaluando variables moderadoras de la relación. Es por esto que el objetivo general del presente estudio fue identificar la existencia de condiciones psicosociales de riesgo para la salud de los académicos de diferentes universidades de Bogotá, y si estas están asociadas con problemáticas de salud tales como síntomas somáticos, ansiedad, depresión y disfunción social. Dados los resultados de trabajos previos se esperaba que dicha relación existiera, en cuyo caso se pretendió determinar si las relaciones entre los FRP y los indicadores de malestar eran 
moderadas por algunas variables demográficas (p. ej., sexo, estado civil, categoría en el escalafón docente, tipo de nombramiento, etc.) y propias del trabajo (p. ej., condiciones del lugar, carga laboral, contenido y características de la tarea, exigencias, papel del académico y desarrollo de la carrera, entre otras).

\section{Método}

Tipo de estudio

La metodología fue predominantemente cuantitativa, pero complementada con estrategias de tipo cualitativo (entrevistas semiestructuradas). En este artículo, solo se presentarán algunos de los datos recogidos mediante encuesta. Con el fin de dar alcance a los objetivos descritos anteriormente, se planteó un diseño predictivo transversal, combinado con un diseño comparativo de grupos naturales (Ato, López, \& Benavente, 2013).

\section{Participantes}

Participaron en total 302 académicos de 21 universidades de la ciudad de Bogotá. Las de mayores porcentajes de docentes en la muestra fueron: la Universidad de los Andes (30.1\%), la Universidad de Bogotá Jorge Tadeo Lozano (19.9 $\%)$, la Universidad Nacional de Colombia (16.2 \%), la Universidad Libre de Colombia (8.3 \%) y la universidad Colegio Mayor de Nuestra Señora del Rosario (5 \%). La edad promedio fue 42.3 años (rango entre 24 y 63), 43.3 para los hombres y 40.7 para las mujeres. Otras características sociodemográficas y laborales de la muestra se presentan en la Tabla 1.

\section{Tabla 1}

Datos sociodemográficos de la muestra

\begin{tabular}{lr}
\hline \multicolumn{1}{c}{ Variable } & \multicolumn{1}{c}{$\%$} \\
\hline Sexo & \\
Hombres & 57.3 \\
Mujeres & 42.7 \\
Estado civil & \\
Casado & 58 \\
Soltero & 24 \\
Divorciado o separado & 11 \\
Otro & 7 \\
Tipo de institución & \\
Pública & 16.9 \\
Privada & 83.1 \\
Nombramiento & \\
Planta & 74.5 \\
Cátedra & 25.5 \\
Categoria & \\
Titular & 8.9 \\
Asociado & 31.8 \\
Asistente & 25.8 \\
Auxiliar & 7.9 \\
Catedrático & 14.2 \\
Ninguna & 11.3 \\
Dedicación por actividad & \\
Docencia & 42.7 \\
Investigación & 24.95 \\
Extensión & 6.92 \\
Tutorías & 10.72 \\
Gestión administrativa & 17.97 \\
Nivel educativo & \\
Doctorado & \\
Máster & \\
Pregrado & \\
Otros & \\
\hline & \\
\hline
\end{tabular}

En promedio, los profesores trabajan con 3.23 grupos $(D E=3.64)$ y 82.97 estudiantes $(D E$ $=58.78)$. Las horas de trabajo semanales son, en promedio, $40.49(\mathrm{DE}=15.73)$ dentro de las cuales están $10.53(D E=8.5)$ horas de clase.

\section{Instrumentos}

Las variables sociodemográficas fueron evaluadas con preguntas desarrolladas por los investigadores específicamente para este estudio. Las demás variables de interés se evaluaron con los cuestionarios estandarizados que se describen a continuación. Entre paréntesis se reportan el número de ítems y los omegas de McDonald de cada escala.

Factores de riesgo psicosocial Job Content Questionnaire (JCQ)

Se utilizó, con algunos pequeños ajustes, la versión en español del JCQ de 27 ítems que 
ha sido empleada y validada previamente con población mexicana (Juárez-García, 2007) y colombiana (Gómez, 2011). Incluye las siguientes escalas: la escala de Control $(\omega=0.82)$, que está compuesta por dos subescalas: Uso de Habilidades (6 ítems, $\omega=0.72$ ) y Toma de Decisiones ( 3 ítems, $\omega=0.77$ ); la escala de Demandas Psicológicas Laborales (5 ítems, $\omega=$ 0.69); la escala de Apoyo del Supervisor (4 ítems, $\omega=0.88$ ); Apoyo de los compañeros (4 ítems, $\omega=0.87$ ); la escala de Inseguridad Laboral (4 ítems, $\omega=0.71$ ) y la de Demandas Físicas del Trabajo (1 ítem). Se calculó un indicador de tensión laboral usando la sugerencia del Centro-JCQ: Un término de razón de tensión laboral: (Demandas *2) / Libertad de decisión. Un puntaje $>1$ podría indicar tensión laboral.

\section{Cuestionario Desequilibrio Esfuerzo-Recompensa} (Effort-Reward Imbalance Questionnaire [ERI]

En Colombia, el instrumento fue validado por Gómez (2010). Esta versión incluye la escala de Esfuerzo extrínseco (6 ítems, $\omega=0.81$ ), la de Recompensa ${ }^{3}$ (11 ítems) y Sobrecompromiso (6 ítems, $\omega=0.76)$. El desequilibrio entre Esfuerzo y Recompensa es una razón que se computa para cada respondiente de acuerdo con un algoritmo predefinido.

Factores psicosociales propios del contexto académico

Para la evaluación de los agentes psicosociales propios y específicos del contexto académico se utilizó la escala diseñada por Silva (2006) la cual contiene una lista de factores agrupados en 7 áreas: a) Condiciones del lugar de trabajo (10 ítems, $\omega=0.78$ ), b) Carga de trabajo (6 ítems, $\omega=0.67)$, c) Contenido y características de la tarea ( 8 ítems, $\omega=0.58$ ), d) Exigencias laborales (7 ítems, $\omega=0.64)$, e) Papel del académico y desarrollo de la carrera ( 7 ítems, $\omega=0.72$ ), f) Interacción social y aspectos organizacionales (9 ítems, $\omega=0.85$ ) y g) Remuneración del rendimiento ( 3 ítems, $\omega=0.52$ ). Se suman los puntajes de cada apartado, y con base en las sumas es posible establecer cuatro niveles de riesgo: nulo, bajo, medio y alto.

\section{Condiciones de malestar 4}

Cuestionario general de salud de Goldberg

Se utilizó el General Health Questionnaire (GHQ-28) de Goldberg (1978) en su adaptación al español de Lobo, Pérez-Echeverría y Artal (1986). El cuestionario cuenta con cuatro factores: Síntomas somáticos $(\omega=0.82)$, Ansiedad $(\omega=0.89)$, Depresión $(\omega=0.89)$ y Disfunción social $(\omega=0.84)$, cada uno con 7 ítems.

\section{Procedimiento}

Se aplicaron los cuestionarios a los docentes de universidades de Bogotá que aceptaron participar voluntariamente en el estudio. De ellos, a 4045 profesores se les invitó a participar mediante un correo electrónico que describía el objetivo del estudio y la forma de participación. Una vez un docente aceptaba (la tasa de participación fue de $7.5 \%$ ), recibía un nuevo correo con el enlace para acceder a la encuesta y contestar mediante el servicio de Survey Monkey; para identificar su cuestionario se le asignó un código. Además, se hizo una cita personal para firmar el consentimiento informado y tomar medidas adicionales no reportadas en este trabajo (tensión arterial, peso y estatura de cada participante).

Se utilizó el programa SPSS versión 18 para realizar los análisis estadísticos. Primero se llevaron a cabo los análisis descriptivos de los FRP y se calcularon diferencias significativas entre ellos de acuerdo con variables sociodemográficas (p. ej., edad, sexo), y propias del trabajo tales como tipo de contrato y categoría docente, usando para ello ANOVAS o chi cuadrado. Posteriormente, se describieron las condiciones de salud y malestar, antes de analizar las relaciones entre cada uno de ellos y los distintos tipos de condiciones psicosociales de trabajo. Finalmente, para cada indicador de malestar se calcularon regresiones por bloques 
con todas las variables que se relacionaron significativamente con él. Los componentes de cada uno de los modelos (JCQ y ERI) se introdujeron en bloques distintos de la regresión para sopesar el aporte relativo de cada uno.

\section{Resultados}

\section{Condiciones psicosociales de trabajo}

Descripción de Factores de Riesgo Psicosocial con el JCQ y el ERI

Los resultados que se presentan en la Tabla 2 muestran los puntajes promedio obtenidos por los participantes en las escalas JCQ y ERI. Comparados con otros grupos ocupacionales evaluados en Colombia (Gómez, 2011), todos los promedios obtenidos indicarían unas condiciones psicosociales laborales significativamente más favorables en términos de mayor uso de habilidades y posibilidad de toma de decisiones (es decir, más control laboral) y menos demandas psicológicas. Lo anterior indica que el nivel de tensión laboral de los profesores universitarios es el más bajo de todos los grupos ocupacionales estudiados hasta ahora en el país (enfermeras, conductores de buses y de transporte intermunicipal, profesores de colegio y grupos de ocupaciones mixtas). Por otro lado, los niveles de apoyo social son comparables a los de otros grupos evaluados en Colombia, pero la inseguridad laboral percibida es una de las más altas (solo superada por la que perciben las enfermeras). Este resultado indicaría que, de acuerdo con el modelo demanda-control, el factor psicosocial de mayor riesgo para los profesores universitarios es la inseguridad laboral.

En el caso de los factores medidos por el ERI, el promedio obtenido en la escala de Esfuerzo es uno de los más altos comparado con los otros grupos ocupacionales evaluados en Colombia (Gómez, 2010), solo superado por el de los profesores de colegio y enfermeras. Sin embargo, en la escala de Sobrecompromiso los promedios obtenidos son comparables con los de otros grupos de trabajadores colombianos, exceptuando nuevamente a los profesores escolares, quienes son los que más se caracterizan por este estilo de afrontamiento en el contexto laboral. Debido a problemas con la calificación de la escala de recompensas, no fue posible comparar la percepción de este factor con otros grupos ocupacionales, ni calcular el desequilibrio entre los esfuerzos y las recompensas percibidos.

\section{Tabla 2}

Valores promedio y desviaciones estándar obtenidos en las escalas del JCQ y del ERI

\begin{tabular}{lrr}
\hline \multicolumn{1}{c}{ Escalas } & $\boldsymbol{M}$ & $\boldsymbol{D E}$ \\
\hline JCQ & & \\
Uso de Habilidades & 40.5 & 5.1 \\
Toma de Decisiones & 39.4 & 7.2 \\
Control & 79.8 & 11.3 \\
Demandas Psicológicas & 30.4 & 6.7 \\
Tensión laboral & 0.8 & 0.2 \\
Apoyo del supervisor & 11.1 & 3 \\
Apoyo de compañeros & 12 & 2.6 \\
Apoyo social & 23.1 & 4.9 \\
Inseguridad laboral & 6.4 & 2.1 \\
ERI & & \\
Esfuerzo & 15.4 & 4.5 \\
Sobrecompromiso & 15.4 & 3.9 \\
\hline
\end{tabular}

Diferencias en los factores psicosociales laborales de acuerdo con variables sociodemográficas y propias del trabajo

La inseguridad laboral percibida por los profesores de las universidades privadas es significativamente más alta, comparada con la de aquellos que trabajan en universidades públicas $(F=22.99 ; p<0.01)$ y la que perciben los profesores de cátedra y auxiliares es más alta que la de académicos titulares, asociados y asistentes. Los profesores de cátedra también perciben mayor inseguridad que los de planta y los que tienen contrato a término fijo más que los que tienen contrato a término indefinido. 
Con respecto a las demandas psicológicas en el trabajo, los profesores de planta son quienes las perciben significativamente más altas que los de cátedra $(F=33.12 ; p<0.01)$. Otras condiciones psicosociales laborales medidas con el JCQ no se diferenciaron entre profesores de diferente tipo de universidad, sexo, tipo de contrato o categoría docente. Finalmente, entre más edad tienen los profesores, menos demandas psicológicas perciben, pero también menos apoyo social por parte de superiores y colegas.

En lo que respecta a las variables medidas por el ERI, tanto el esfuerzo extrínseco $(F=10.56$; $p<0.01)$ como el intrínseco (sobrecompromiso) $(F=11.77 ; p<0.01)$ que las profesoras perciben invertidos en el trabajo son significativamente más altos que los de sus colegas masculinos. Las mismas variables diferencian a los profesores de planta y los de cátedra, percibiendo los primeros más esfuerzo $(F=18.22 ; p<0.01)$ y sobrecompromiso $(F=6.61 ; p<0.01)$ con su trabajo.

Factores psicosociales característicos del ámbito académico

En la Tabla 3, se exponen los promedios obtenidos en cada uno de los factores psicosociales propios del trabajo académico que indican la frecuencia con la que se presentan estas condiciones en el ámbito laboral docente. En primera instancia, los resultados obtenidos en las Condiciones del lugar de trabajo, el Contenido y características de la tarea, el Papel del académico y desarrollo de la carrera, la Interacción social y la Remuneración revelan que la frecuencia con que se presentan aspectos negativos en estos factores es baja, por lo tanto, no se consideran riesgosos para la población evaluada. Dimensiones como las Exigencias laborales y la Carga de trabajo son factores valorados en riesgo medio debido al tipo de condiciones negativas medidas por estas escalas (que corresponden conceptualmente a lo que evalúa la escala de demandas psicológicas laborales del JCQ, pero en este caso se evalúan condiciones específicas del trabajo de un académico universitario). Este resultado indica que los profesores están percibiendo que su carga laboral es excesivamente alta (en términos de tiempo para realizarla, número de tareas por realizar, exigencias físicas y mentales que las tareas imponen) y por tanto puede representar un riesgo para su salud.

\section{Tabla 3}

Descriptivos de los factores psicosociales del trabajo académico

\begin{tabular}{lrrcrr}
\hline \multicolumn{1}{c}{ Factores } & $\boldsymbol{M}$ & $\boldsymbol{D E}$ & Riesgo* $^{\text {* }}$ & $\begin{array}{c}\text { \% } \\
\text { Nulo-Bajo }\end{array}$ & $\begin{array}{c}\text { \% } \\
\text { Medio-Alto }\end{array}$ \\
\hline Condiciones del lugar de trabajo & 11 & 6.43 & Bajo & 80 & 20 \\
Carga de trabajo & 14 & 4.07 & Medio & 16.4 & 83.6 \\
Contenido y caracteristicas de la tarea & 9 & 4.2 & Bajo & 85 & 15 \\
Exigencias laborales & 14 & 4.96 & Medio & 30.3 & 69.7 \\
Papel del académico & 7 & 4.64 & Bajo & 83.9 & 16.1 \\
Interacción social & 10 & 6.43 & Bajo & 95.6 & 4.4 \\
Remuneración & 6 & 2.43 & Bajo & 61.1 & 38.9 \\
\hline \multicolumn{5}{c}{ * Riesgo promedio de toda la muestra. }
\end{tabular}

Diferencias en los factores psicosociales laborales propios del ámbito académico de acuerdo con variables sociodemográficas y propias del trabajo

Para cumplir el supuesto de la prueba chi cuadrado, donde ninguna casilla debe tener menos de cinco observaciones, se recodificaron y agruparon las respuestas de los docentes en dos niveles de riesgo (nulo-bajo y medio-alto).

Se encontró una diferencia estadísticamente significativa en las Condiciones de trabajo por Tipo de universidad $\left(\chi^{2}(3, N=275)=6.5, p=\right.$ $0.01)$ que indica que existe una mayor proporción de docentes de universidades privadas (82.7 $\%)$ que de docentes de universidades públicas $(65.9 \%)$ que perciben como poco riesgosas las condiciones físicas del lugar de trabajo. También se encontraron diferencias en la percepción de la Remuneración de acuerdo con el Tipo de contrato $\left(\chi^{2}(3, N=275)=6.66, p=0.01\right)$, de donde se puede inferir que para los docentes con contrato a término indefinido $(72.8 \%)$ la remuneración no representa un riesgo psicosocial comparados con los docentes con contrato a término fijo (56.2\%). En la Tabla 4, se presentan las diferencias significativas relacionadas con la percepción de alta carga de trabajo y mayores exigencias laborales. 
Tabla 4

Diferencias en carga de trabajo y exigencias

laborales percibidas, de acuerdo con variables sociodemográficas.

\begin{tabular}{|c|c|c|c|c|}
\hline Carga de trabajo & $\%$ & Exigencias laborales & $\%$ & Diferencias \\
\hline Planta & 94.1 & Cátedra & 53.5 & $x^{2}(3, N=275)=63.42, p=0$ \\
\hline Término indefinido & 92.6 & Término fijo & 79.9 & $x^{2}(3, N=275)=6.73, p=0$ \\
\hline Pública & 95.5 & Privada & 81.4 & $x^{2}(3, N=275)=5.34, p=0.02$ \\
\hline Mujeres & 77.3 & Hombres & 64.6 & $x^{2}(3, N=274)=4.98, p=0.02$ \\
\hline Planta & 77 & Cátedra & 48.6 & $x^{2}(3, N=274)=19.89, p=0$ \\
\hline \multirow{5}{*}{ Asistentes } & \multirow{5}{*}{81.2} & Titulares & 58.3 & \multirow{5}{*}{$x^{2}(5, N=274)=18.66, p=0$} \\
\hline & & Asociados & 75.3 & \\
\hline & & Auxiliares & 76.2 & \\
\hline & & Catedráticos & 46.3 & \\
\hline & & No categorizados & 63.3 & \\
\hline Tiempo completo & \multirow[t]{2}{*}{77} & Medio tiempo & $\begin{array}{l}55.6 \\
564\end{array}$ & $x^{2}(6, N=274)=12.68, p=0$ \\
\hline \multirow{3}{*}{ Doctorado } & & Pregrado & 58.8 & \multirow{3}{*}{$x^{2}(9, N=274)=8.34, p=0.03$} \\
\hline & \multirow{2}{*}{81.5} & Maestría & 66.4 & \\
\hline & & $\begin{array}{l}\text { Otro nivel de } \\
\text { escolaridad }\end{array}$ & 60 & \\
\hline
\end{tabular}

Indicadores de malestar psicológico

Los promedios obtenidos en el cuestionario de salud de Goldberg, el cual evalúa la frecuencia con que se experimentaron en las últimas semanas síntomas de ansiedad $(M=13.09 ; D E$ $=4.78)$, depresión $(M=8.48 \mathrm{DE}=2.87)$, síntomas psicosomáticos $(\mathrm{M}=13.98 ; \mathrm{DE}=$ 4.2) y ajuste social $(M=16.38 ; D E=3.73)$, indican que, en las últimas semanas, los docentes no experimentaron esta sintomatología más de lo habitual, exceptuando indicadores de ajuste social tales como problemas para sentir que se están haciendo las cosas bien, para mantenerse ocupado y para sentir que están disfrutando de sus actividades del día a día, los cuales se están percibiendo un poco más de lo habitual.

Relación entre las condiciones psicosociales laborales y los indicadores de malestar psicológico

Las correlaciones entre factores psicosociales laborales con ansiedad y síntomas psicosomáticos son, sin excepción, significativas, tal como puede observarse en la Tabla 5. Se destaca que mayores demandas, menor control, menos apoyo social, más inseguridad laboral, mayor esfuerzo invertido en el trabajo y mayor característica de sobrecompromiso se relacionan con mayor ansiedad y síntomas psicosomáticos. La depresión, por su parte, es mayor cuando se percibe menos control laboral y hay mayor percepción de esfuerzo tanto intrínseco como extrínseco. Finalmente, se presentan mayores indicadores de desajuste social cuando se perciben menores niveles de control laboral y mayores niveles de sobre-compromiso con el trabajo.

\section{Tabla 5}

Correlaciones entre los factores medidos por los cuestionarios ERI y JCQ con los indicadores de malestar

\begin{tabular}{|c|c|c|c|c|}
\hline $\begin{array}{c}\text { Escalas del GHQ } \\
\text { Escalas ERI y JCQ }\end{array}$ & $\begin{array}{c}\text { Sintomas } \\
\text { Psicosomáticos }\end{array}$ & $\begin{array}{c}\text { Ansiedad y } \\
\text { problemas de } \\
\text { sueño }\end{array}$ & Depresión & $\begin{array}{l}\text { Ajuste } \\
\text { social }\end{array}$ \\
\hline Esfuerzo & $0.41^{* \prime}$ & $0.39^{* *}$ & $0.15^{\circ}$ & 0.09 \\
\hline Sobrecompromiso & $0.41^{* *}$ & $0.52^{* *}$ & $0.22^{* *}$ & $0.18^{* *}$ \\
\hline Uso de habilidades & $-0.22^{*}$ & $-0.25^{*}$ & $-0.30^{* *}$ & $-0.19^{* *}$ \\
\hline Toma de Decisiones & $-0.29^{* \prime}$ & $-0.32^{* *}$ & $-0.29^{* *}$ & $-0.19^{* *}$ \\
\hline Control & $-0.29^{* *}$ & $-0.32^{*}$ & $-0.33^{* \prime}$ & $-0.21^{* *}$ \\
\hline Demandas Psicológicas & $0.31^{*}$ & $0.33^{*}$ & 0.11 & 0.04 \\
\hline Apoyo Supervisor & $-0.25^{\prime \prime}$ & $-0.24^{* \prime}$ & $-0.17^{* \prime}$ & $-0.13^{\circ}$ \\
\hline Apoyo Compañeros & $-0.2^{* *}$ & $-0.22^{*}$ & $-0.2^{* \prime}$ & -0.05 \\
\hline Inseguridad Laboral & $0.17^{* \prime}$ & $0.18^{* \prime}$ & 0.07 & 0.09 \\
\hline Apoyo Social & $-0.26^{*}$ & $-0.26^{* *}$ & $-0.21^{*}$ & -0.1 \\
\hline Tensión laboral & $0.37^{* *}$ & $0.39^{* *}$ & $0.23^{* *}$ & 0.11 \\
\hline \multicolumn{5}{|c|}{$\begin{array}{l}\text { ** La correlación es significativa } \\
\text { al nivel } 0.01 \text { (bilateral). } \\
\text { *La correlación es significante } \\
\text { al nivel } 0.05 \text { (bilateral). }\end{array}$} \\
\hline
\end{tabular}

Para valorar el peso relativo de los diferentes factores psicosociales laborales asociados con cada uno de los indicadores de malestar, se realizaron regresiones lineales por bloques, donde se controló la edad cuando esta se asoció con alguno de los indicadores de malestar. Adicionalmente, los componentes de cada uno de los modelos de factores psicosociales laborales se introdujeron en bloques distintos de la regresión, para evaluar la capacidad predictiva de los componentes de cada modelo por separado.

En el caso de los síntomas psicosomáticos, el control laboral, la inseguridad laboral, el esfuerzo y el sobrecompromiso explicaron significativamente casi el $25 \%$ de la varianza de la percepción de síntomas psicosomáticos $(F=6.803, p<0.01)$, mostrando que a más control laboral $(\mathrm{B}=-0.5)$ hay menos percepción de síntomas psicosomáticos; además, una mayor percepción de esfuerzos tanto extrínsecos $(\mathrm{B}=$ 0.174) como intrínsecos $(\mathrm{B}=0.252)$ y mayor percepción de inseguridad laboral $(B=0.086)$ permiten predecir un aumento en la percepción de esta sintomatología.

En el caso de la ansiedad, el control laboral (B $=-0.86)$ y la inseguridad $(\mathrm{B}=0.242)$, además del esfuerzo $(\mathrm{B}=0.129)$ y el sobrecompromiso 
$(\mathrm{B}=0.49)$ explicaron significativamente el 32 $\%$ de la varianza $(F=19.373, p<0.01)$ La depresión se evaluó frente al control, el apoyo social, el esfuerzo y el sobrecompromiso. En este caso, el $12 \%$ de la varianza $(F=9.203, p<$ 0.01) fue explicado por el control $(B=-0.075)$ y el sobrecompromiso $(B=0.118)$; ninguna de las otras variables fue significativa. Finalmente, el ajuste social se evaluó frente al control laboral, el apoyo del supervisor y el sobrecompromiso encontrándose que el $6.8 \%$ de la varianza $(F=$ $5.788, p<0.01)$ se explica por la edad, el control $(\mathrm{B}=-0.064)$ y el sobrecompromiso $(\mathrm{B}=0.132)$.

En resumen, algunos factores psicosociales parecen estar más asociados con los indicadores de malestar que otros. El control laboral y el sobrecompromiso se repiten una y otra vez como factores predictores de diferentes indicadores de malestar psicológico. Además, demasiados esfuerzos y la alta percepción de inseguridad laboral se asocian con mayor frecuencia en el reporte de síntomas psicosomáticos y más ansiedad.

\section{Discusión}

Entre los FPR señalados consistentemente por los profesores universitarios como estresores potenciales están las excesivas exigencias laborales y la alta carga de trabajo, lo cual también coincide con estudios previos realizados en varios países (García et al., 2016; Keser et al., 2019; Kinman, 2001; Kinman et al., 2006; Pando, Castañeda et al., 2006). Los resultados del presente trabajo confirman que quienes más perciben demandas y carga de trabajo son también quienes más reportan problemas de malestar de diverso tipo, tales como ansiedad, síntomas psicosomáticos y desajuste social. Se encontró que las demandas, exigencias y esfuerzos laborales son percibidos como más altos por parte de los profesores más jóvenes, con categoría de asistentes, todo lo cual coincide con los resultados de estudios previos (p. ej., Cifre \& Llorens, 2001); por ejemplo, los ingleses (problemas de comunicación, falta de apoyo para las actividades académicas, responsabilidades administrativas onerosas, sobrecarga de información, enseñanza a muchos estudiantes y realizar investigación de calidad con recursos limitados) (Kinman et al., 2006), así como los latinoamericanos (tener que realizar diferentes tipos de tareas: docencia, investigación, consultoría y gestión) (Pando, Aranda et al., 2006; Pando, Castañeda et al., 2006). Al parecer, las condiciones laborales de los profesores en las universidades en Bogotá son muy similares a las que se han evaluado en otras partes del mundo.

La inseguridad laboral también parece ser un FRP para los profesores universitarios en la muestra estudiada en Bogotá. Este resultado coincide con lo reportado en varios estudios en el Reino Unido y en Australia (Kinman et al., 2006; Winefield et al., 2003). Si tenemos en cuenta que la percepción de inseguridad laboral en Colombia es de las más altas según los países del mundo que la han evaluado (Gómez, 2010; Gómez \& Perilla, 2011), podemos afirmar que este factor de riesgo es uno de los más importantes, especialmente para los profesores más jóvenes.

La percepción de inseguridad laboral parece, en este caso, ir atada al tipo de contratos que se realizan con la mayor parte de los profesores (contratos a término fijo) y la evaluación del desempeño que puede poner en riesgo la continuidad del contrato. Este resultado coincide con el de varios de los trabajos citados en este artículo, lo que indica que esta es una problemática que afecta a los profesores universitarios de manera similar en varios países tan disímiles como Colombia y Australia. La inseguridad laboral es un fenómeno subjetivo, basado en las percepciones e interpretaciones del ambiente laboral inmediato (Sverke \& Hellgren, 2002), y se refiere a la expectativa de los trabajadores sobre la continuidad de su condición y a una preocupación general por la percepción de amenazas a ella. Como fuente de malestar de los trabajadores, recientemente empezó a generar el interés de los investigadores, con motivo de los cambios globales en la naturaleza de la organización laboral que busca disminuir costos además de mejorar la efectividad y competitividad organizacional, 
mediante procesos de reducción de sus plantas de personal y aumento de la tecnología para atender procesos productivos, así como la flexibilización de la legislación laboral en la que los contratos temporales o a término fijo son ahora la regla y no la excepción. Los resultados de este y otros estudios indican que además del tipo de contrato, en el caso de los profesores la inseguridad laboral está estimulada por la falta de certeza acerca de los requisitos que deben reunir para obtener una mayor garantía de continuidad, que según los profesores, está demasiado centrada en que se demuestre la investigación y especialmente en publicaciones en revistas de alto nivel (Gómez, 2012; Gómez \& Perilla, 2011). Al parecer los académicos tienen la impresión de que hay otras tareas que interfieren con el tiempo y las energías necesarias para producir este tipo de publicaciones, y que no todos se sienten del todo preparados para cumplir con este propósito y tampoco están percibiendo el apoyo institucional necesario para lograrlo.

Por su parte, la remuneración no parece constituir, para la mayor parte de los participantes, una fuente de riesgo psicosocial en el entorno laboral académico. Es posible que este resultado se deba a las diferencias en remuneración que caracterizan las instituciones participantes. Hay algunas universidades, entre las que se encuentran la Universidad Nacional y la de los Andes, que han hecho esfuerzos importantes por mejorar las remuneraciones de los docentes. Sin embargo, este es un factor con diferencias importantes entre las instituciones de educación superior. Otra razón puede ser que la muestra estuvo compuesta, en su mayor parte, por profesores de planta y no de cátedra. Son estos últimos los que presentan más riesgo al respecto, debido en parte a que sus contratos son de menor duración y a que las instituciones pagan un valor por hora menor al que le pagan a sus profesores más estables.

La evaluación de qué tanto las relaciones entre las condiciones laborales y las de salud variaron en función de variables demográficas y asociadas al trabajo indicó que la inseguridad laboral percibida es mayor entre los profesores de universidades privadas, los más jóvenes, con títulos de asistentes o auxiliares o con contrato de cátedra o a término fijo. Claramente, aquellos profesores cuyos contratos son menos estables, son renovados cada cuatro, cinco o seis meses (profesores de cátedra), o cuya renovación cada año depende de los logros que demuestren (los jóvenes y asistentes, o que trabajan en universidades privadas que tienen contratos laborales a término fijo más frecuentemente que las universidades públicas), son los que más están percibiendo que su trabajo está en riesgo. En muchas universidades, adquirir el estatus de profesor asociado o titular representa estabilidad y permanencia.

Los resultados de este estudio señalan la necesidad para las universidades de revisar cuáles son sus prioridades, de definir cómo aprovechar de la mejor manera el potencial de su principal capital que son los académicos, sin que estos terminen lastimados por sus esfuerzos y compromiso con las instituciones. A propósito de esta reflexión y de estos resultados, Boyer (1990) se pregunta cómo es posible hablar de mejorar la calidad de la educación si no se reconoce el tiempo que los académicos dedican a enseñar. En el ambiente actual, los estudiantes son los perdedores, según el autor. Las universidades reclutan agresivamente estudiantes para sus programas de pregrado, algunas argumentando como atractivo la calidad de la docencia y el espíritu de comunidad que se ofrece, pero la realidad es que en la mayor parte de las instituciones universitarias la buena docencia no se premia, no se considera dentro de las prioridades para ascender en la carrera profesoral, y aquellos que le dedican mucho tiempo a este tipo de tareas ponen en riesgo su promoción dentro de la carrera y, por tanto, su estabilidad laboral.

La investigación es fundamental, pero las universidades necesitan promover de manera real el compromiso de sus académicos con la docencia y el servicio. Boyer (1990) afirma que es tiempo de reconocer el rango completo de los talentos de los académicos y la gran diversidad de funciones que la educación superior debe cumplir. Sin embargo, agregamos, quizá no todos los profesores deban realizar todo el rango de 
tareas ni todas las universidades deban trabajar por lo mismo. En este sentido, un exrector de la Universidad de los Andes propuso hace algunos años que "se logre en Colombia la focalización de las Instituciones de Educación Superior en universidades de investigación, universidades de pregrado y posgrado, universidades de pregrado, "community colleges", instituciones tecnológicas e instituciones técnicas, las IES podrían ser más efectivas y se lograrían mayores beneficios para las regiones" (Angulo, 2011). Una posible consecuencia de esta especialización sería que los profesores pudieran dedicarse más a unas tareas que a otras, dependiendo de sus gustos y habilidades, y que no deban atender toda clase de actividades como lo están haciendo hoy, lo cual, según lo que aquí se reporta, se constituye en un importante FRP, como también lo señalan Padilla y Thompson (2016) para el caso estadounidense.

Para terminar, Boyer (1990) afirma que es tiempo de que las universidades se midan a sí mismas, más que por el estatus externo, por los valores determinados por su propia misión distintiva. Solo así, se logrará tener educación realmente de calidad, que se reconozca y se recompense, y quizá, sugerimos, también un cuerpo profesoral más sano y satisfecho.

Esta investigación, a diferencia de otras realizadas previamente en Latinoamérica y, particularmente, en Colombia, no solo confirmó que hay condiciones psicosociales de trabajo que ponen en riesgo el bienestar de los profesores universitarios, tal como está ocurriendo en otros países; también identificó que estas condiciones efectivamente están asociadas con mayores niveles de malestar y que dicha relación está moderada por variables sociodemográficas (p. ej., edad, sexo) y propias del trabajo, tales como el clase de contrato, la categoría profesoral y el tipo de universidad. Este estudio evaluó los FRP descritos por los modelos de estrés laboral más estudiados en la literatura, lo que permite comparar a los profesores universitarios con otros grupos ocupacionales colombianos y con docentes de otras universidades del mundo. Los factores psicosociales evaluados por los modelos Demanda-control y ERI han demostrado ampliamente la asociación positiva con múltiples problemas de salud física y mental. Este estudio confirmó algunas de ellas y otras más (Gómez, 2012), aunque es necesario continuar evaluando otras problemáticas de salud distintas a las reportadas en este trabajo.

\section{Limitaciones del estudio}

Dado que los datos de la presente investigación no fueron tomados de manera longitudinal, no es posible hacer atribuciones de causalidad en el sentido de que son las condiciones psicosociales laborales las que están generando los problemas de salud reportados. Otra interpretación posible de las asociaciones halladas es que los profesores con más problemas de salud perciben condiciones psicosociales de trabajo más negativas. Sin embargo, en el contexto de lo que algunos estudios longitudinales sobre condiciones psicosociales laborales y su impacto en la salud (Schnall, Dobson, \& Rosskam, 2011) han mostrado, es posible afirmar que la primera opción causal es más probable que la segunda.

Por otro lado, el tamaño de la muestra estudiada, a pesar de no ser pequeño, no es representativo de la población de profesores universitarios existente en Bogotá. Las condiciones psicosociales laborales pueden ser aún más difíciles, o mejores, que las que aquí se señalan. $\mathrm{Al}$ respecto, es interesante destacar que la invitación a participar se pudo hacer fundamentalmente a profesores de instituciones que tienen disponibles sus direcciones de correo electrónico en su web. De esa manera, se pudo enviar una invitación directa a la gran mayoría. Cuando esto no fue posible, y los intentos de comunicación con los profesores debieron hacerse a través de oficinas institucionales, en su mayor parte vicerrectorías académicas u oficinas de recursos humanos, ningún profesor respondió a la invitación, sea porque no la recibieron o porque el hecho de recibir una invitación a través de responsables de la institución para evaluar condiciones psicosociales de trabajo, inducía a algún tipo de temor o recelo con respecto a la confidencialidad de la información. 
Otra probable limitación es que los participantes podrían haberse autoseleccionado de manera sesgada. No es descartable que la invitación haya sido aceptada mayoritariamente por aquellos docentes que tienen una percepción más negativa de sus condiciones laborales, y por tanto la participación en este proyecto sería una manera de hacer visible su malestar. Sin embargo, también es probable que la autoselección haya sido, al contrario: las personas con condiciones psicosociales laborales más negativas no aceptan la invitación precisamente porque estas limitan su tiempo y energía para participar en una actividad voluntaria de investigación.

Finalmente, sería importante llevar a cabo investigaciones longitudinales donde se estudien a mediano y largo plazo las consecuencias de los FRP en la salud de los profesores universitarios, pues se podrían establecer relaciones de causalidad, lo cual tal vez ayudaría a disponer de mayor evidencia para confirmar los resultados que se presentan en este trabajo.

\section{Referencias}

Angulo, C. (17 de octubre de 2011). El proyecto de reforma Oportunidad para hablar de calidad de la educación. El Tiempo.com. Recuperado de https://www.eltiempo.com/ archivo/documento/MAM-4899872

Ato, M., López, J., \& Benavente, A. (2013). Un sistema de clasificación de los diseños de investigación en psicología. Anales de Psicología, 29(3), 1038-1059. https://doi.org /10.6018/analesps.29.3.178511

Boyer, E. L. (1990). Scholarship reconsidered. Priorities of the professoriate. Princeton, NJ: The Carnegie Foundation for the Advancement of Teaching.

Cifre, E., \& Llorens, S. (2001). Burnout en profesores de la UJI: un estudio diferencial. Fòrum de Recerca, 7, 2-11. Recuperado de ht tp://repositori.uji.es/xmlui/bitstream/handl e/10234/79710/forum_2001_15.pdf?seque nce $=1$

Collado, P., Soria, C., Canafoglia, E., \& Collado, S. (2016). Condiciones de trabajo y salud en docentes universitarios y de enseñanza media de Mendoza, Argentina: entre el compromiso y el desgaste emocional. Salud Colectiva, 12 (2), 203-220. https://doi.org/1 $0.18294 / \mathrm{sc} .2016 .710$

Cross, G., \& Carroll, D. (1990). Goodwill under stress: Morale in UK universities. Londres: Association of University Teachers.

Doménech, B. (1995). Introducción al síndrome "burnout" en profesores y maestros y su abordaje terapéutico. Psicología Educativa, 1, 63-78. Recuperado de https://dialnet.uni rioja.es/servlet/articulo?codigo $=2826433$

Doyle, C., \& Hind, P. (2002). Occupational stress, burnout and job status in female academics. Gender, Work and Organization, 5(2), 67-82. https://doi.org/10.1111/1468-0 432.00047

García, M. M., Iglesias, S., Saleta, M., \& Romay, J. (2016). Riesgos psicosociales en el profesorado de enseñanza universitaria: diagnóstico y prevención. Journal of Work and Organizational Psychology, 32, 173-182. https://doi.org/10.1016/j.rpto.2016.07.001

Gómez, V. (2010). Assessment of psychosocial stressor at work: Psychometric properties of the Spanish version of the ERI (Effort-Reward Imbalance Questionnaire) in Colombian workers. Revista de Psicología del Trabajo y de las Organizaciones, 26(2), 147-156. https://doi.org/10.5093/tr2010v2 $6 \mathrm{n} 2 \mathrm{a} 6$

Gómez, V. (2011). Assessment of psychosocial stressor at work: Psychometric properties of the Spanish version of the JCQ in Colombian workers. Revista Latinoamericana de Psicología, 43(2), 125-138. Recuperado de http://www.scielo.org.co/scielo.php?scri pt $=$ sci_arttext\&pid $=$ S0120-05342011000 200012

Gómez, V. (2012). Riesgos para la salud de profesores universitarios derivados de factores psicosociales-laborales (Documento CESO 196). Bogotá: Ediciones Uniandes.

Gómez, V., \& Perilla, L. (2011). Tensión laboral en varios grupos ocupacionales colombianos: validación del modelo 
demanda-control y del instrumento Job Content Questionnaire (JCQ). Ciencia $\mathcal{E}$ Trabajo, 13(42), 208-216.

Heredia, S., Morales, M. F., Infante, R., Sánchez, D., Páez, C., \& Gabini, S. (2018). Psychosocial risk factors in university teachers. Revista Espacios, 39(49). Recuperado de http://www.revistaespacios. com/a18v39n49/a18v39n49p18.pdf

Instituto Internacional de la Unesco para la Educación Superior en América Latina y el Caribe. (2018). La educación superior, internacionalización e integración en América Latina y el Caribe. Balance regional y prospectiva. Córdoba, AR: Universidad Nacional de Córdoba.

Jackson, C., \& Hayday, S. (1997). Staff attitudes at the University of Central Lancashire. Brighton: Institute for Employment Studies.

Johnson, J., Hall, E., \& Theorell, T. (1989). The combined effects of job strain and social isolation on the prevalence and mortality incidence of cardiovascular disease in a random sample of the Swedish male working population. Scandinavian Journal of Work and Environmental Health, 15, 271-279. https://www.ncbi.nlm.nih.gov/pu bmed/2772582

Juárez-García, A. (2007). Factores psicosociales laborales relacionados con la tensión arterial y síntomas cardiovasculares en personal de enfermería en México. Salud Pública de México, 49(2), 109-117. Recuperado de https://pdfs.semanticscholar.org/2909/d de3fca62d53bae6b487a8f883d8e83a4721. pdf

Karasek, R. A., \& Theorell, T. (1990). Healthy work: Stress, productivity and the reconstruction of working life. Nueva York: Basic Books.

Keser, A., Li, J., \& Siegrist, J. (2019). Examining Effort-Reward Imbalance and depressive symptoms among Turkish university workers. Workplace Health $\mathcal{B}$ Safety, 67(3), 131-136. https://doi.org/10.1 177/2165079918807227
Kinman, G. (1998). Pressure points: A survey into the causes and consequences of occupational stress in UK academic and related staff. Londres: Association of University Teachers.

Kinman, G. (2001). Pressure points: A review of research on stressors and strains in UK academics. Educational Psychology, 21(4), 473-492. https://doi.org/10.1080/01443410 120090849

Kinman, G., \& Jones, F. (2003). 'Running up the down escalator': Stressors and strains in UK academics. Quality in Higher Education, 9(1), 21-38. https://doi.org/10.1080/13538 320308162

Kinman, G., Jones, F., \& Kinman, R. (2006). The well-being of the UK academy, 1998-2004. Quality in Higher Education, 12 (1), 15-27. ht tps://doi.org/10.1080/13538320600685081

Lemos, M., Calle, G., Roldán, T., Valencia, M., Orejuela, J. J., \& Román-Calderón, J. P. (2019). Factores psicosociales asociados al estrés en profesores universitarios colombianos. Diversitas: Perspectivas en Psicología, 15(1), 61-72. https://doi.org/10.1 5332/s1794-9998.2019.0015.05

Lobo, A., Pérez-Echevarría, M. J., \& Artal, J. (1986). Validity of the scaled General Health Questionnaire (GHQ-28) in a Spanish population. Psychological Medicine, 16, 135-140. https://doi.org/10.1017/s0033 291700002579

Mark, G., \& Smith, A. (2012). Effects of occupational stress, job characteristics, coping, and attributional style on the mental health and job satisfaction of university employees. Anxiety, Stress, $\mathcal{E}^{2}$ Coping, 25(1), 63-78. https://doi.org/10.108 0/10615806.2010.548088

McClenahan, C., Giles, M., \& Mallett, J. (2007). The importance of context specificity in work stress research: A test of the DemandControl-Support model in academics. Work E3 Stress, 21(1), 85-95. https://doi.org/10.1 080/02678370701264552

McInnis, C. (1999). The work roles of academics in Australian universities. Melbourne: 
Department of Education, Training and Youth Affairs.

Padilla, M., \& Thompson, J. (2016). Burning out faculty at doctoral research universities. Stress and Health, 32, 551-558. https://doi.o $\mathrm{rg} / 10.1002 / \mathrm{smi} .2661$

Pando, M., Aranda, C., Aldrete, M. G., Flores, E., \& Pozos, E. (2006). Factores psicosociales y burnout en docentes del Centro Universitario de Ciencias de la Salud. Investigación en Salud, 8(3), 173-177. http://www.medigraphic.com/pdf s/invsal/isg-2006/isg063f.pdf

Pando, M., Castañeda, J., Gregoris, M., Aguila, A., Ocampo, L., \& Navarrete, R. M. (2006). Factores psicosociales y síndrome de burnout en docentes de la Universidad del Valle de Atemajac, Guadalajara, México. Salud en Tabasco, 12 (3), 523-529. http://www.redalyc.org/como citar.oa?id $=48712304$

Pujol-Cols, L., \& Arraigada, M. (2017). Propiedades psicométricas del Cuestionario de Riesgos Psicosociales Copsoq-Istas 21 y aplicación en docentes universitarios argentinos. Cuadernos de Administración, 30(55), 97-125. https://doi.org/10.11144/Ja veriana.cao30-55.ppcr

Resolución 2646 de 2008 [Ministerio de la Protección Social]. Por la cual se establecen disposiciones y se definen responsabilidades para la identificación, evaluación, prevención, intervención y monitoreo permanente de la exposición a factores de riesgo psicosocial en el trabajo y para la determinación del origen de las patologías causadas por el estrés ocupacional. Julio 17 de 2008.

Rodríguez, M., Orozco, M., Aguilar, M., Báez, M., Herrera, M., \& Méndez, A. (2018). Factores psicosociales y estrategias de afrontamiento asociadas al estrés en profesores universitarios. Revista de la Asociación Española de Especialistas en Medicina del Trabajo, 27(4), 197-203. Recuperado de http://scielo.isciii.es/scielo.php?script $=$ s ci_arttext\&pid $=$ S1132-625520180004000 02

Sauter, S. L., Murphy, L. R., Hurrell, J., \& Levi, L. (1998). Factores psicosociales y de organización. En J. Mager Stellman (Ed.), Enciclopedia de salud y seguridad en el trabajo (pp. 34.2-34.3) Madrid: Ministerio de Trabajo y Asuntos Sociales.

Schnall, P., \& Belkic, K. (2000). Point estimates of blood pressure at the worksite. En P. Schnall, K. Belkic, P. Landsbergis \& D. Baker (Eds.), The workplace and cardiovascular disease. Occupational Medicine (pp. 203-208). Philadelphia: Hanley \& Belfus.

Schnall, P., Dobson, M., \& Rosskam, E. (2011). Trabajo no saludable. Causas, consecuencias y curas. Bogotá: Ediciones Uniandes.

Siegrist, J. (2002). Effort-Reward Imbalance at work and health. En P. Perrewe \& D. Ganster (Eds.), Research in occupational stress and well-being. Vol. 2: Historical and current perspectives on stress and health (pp. 261-291). Nueva York: JAI Elsevier.

Silva, N. (2006). Factores psicosociales y desgaste profesional en académicos del Centro Universitario de Ciencias Biológicas Agropecuarias (Tesis doctoral inédita). Universidad de Guadalajara, México.

Sverke, M., \& Hellgren, J. (2002). The nature of job insecurity: Understanding employment uncertainty on the brink of a new millennium. Applied Psychology: An International Review, 51 (1), 23-42. https://d oi.org/10.1111/1464-0597.0077z

Theorell, T., \& Johnson, J. (1998). Enfermedades cardiovasculares. En J. Mager Stellman (Ed.), Enciclopedia de salud y seguridad en el trabajo (pp. 34.67-34.68). Madrid: OIT.

Unda, S., Uribe, F., Jurado, S., García, M., Tovalín, H., \& Juárez, A. (2016). Elaboración de una escala para valorar los factores de riesgo psicosocial en el trabajo de profesores universitarios. Revista de Psicología del Trabajo y de las Organizaciones, 32 (2), 67-74. https://doi.org/10.1016/j.rpto .2016 .04 .004 
Van Der Doef, M., \& Maes, S. (1999). The Job Demand-Control (support) model and psychological well-being: A review of 20 years of empirical research. Work and Stress, 13(2), 87-114. https://doi.org/10.1080/026 783799296084

Winefield, A., Gillespie, N., Stough, C., Dua, J., Hapuarachchi, J., \& Boyd, C. (2003). Occupational stress in Australian university staff: Results from a national survey. International Journal of Stress Management, 10(1), 51-63. https://doi.org/10.1037/10725245.10.1.51

Winefield, A., \& Jarrett, R. (2001). Occupational stress in university staff. International Journal of Stress Management, 8(4), 285-298. https: //doi.org/10.1023/A:1017513615819

\section{Notas}

* Artículo de investigación.

1 Los componentes más importantes de este modelo son las demandas psicológicas de la labor y el control. Según el modelo, el factor primordial de riesgo psicosocial relacionado con el trabajo es la falta de control sobre cómo alguien cumple con sus demandas y cómo usa sus habilidades, partiendo de la hipótesis de que los trabajos que probablemente causan más malestar son aquellos en los que los trabajadores se enfrentan a altas demandas y bajo control (esta combinación se ha llamado "alta tensión laboral"). El apoyo social en el lugar de trabajo por parte de supervisores o de compañeros y la inseguridad laboral son otros factores propuestos por el modelo, que, bajo las actuales condiciones de empleo, son de importancia creciente. El primero se refiere a niveles globales de interacción social servicial disponible, que podría afectar el bienestar de los trabajadores actuando como un amortiguador entre los estresores psicosociales y los resultados adversos a la salud. "La peor combinación -altas demandas, baja libertad de decisión y bajo apoyo- tendrá las consecuencias más adversas" (Johnson, Hall, \& Theorell, 1989, p. 207).
2 El modelo propone que las experiencias estresantes en el trabajo y los efectos negativos en la salud derivados de ellas resultan de una percepción de desequilibrio entre los esfuerzos invertidos en el trabajo y las recompensas obtenidas. Las ganancias o recompensas se distribuyen a los trabajadores a través de tres elementos: dinero, estima y control del estatus laboral en términos de tener prospectos de promoción y seguridad laboral. La combinación de percepción del desequilibrio mencionado con un excesivo nivel de compromiso con el trabajo (esfuerzo intrínseco o sobrecompromiso), incrementa la probabilidad de que los empleados presenten activación psicobiológica sostenida y que esta, a su vez, se asocie con reacciones de estrés y problemas de salud.

3 Debido a problemas con la calificación de la escala, no es posible reportar datos de confiabilidad.

4 En la investigación, se evaluaron otros indicadores de salud y malestar que no se reportan aquí por razones de espacio. Estos fueron: burnout, percepción del estado de salud, problemas de salud diagnosticados, percepción sobre los cambios en la salud en los últimos años y tensión arterial (Gómez, 2012). 